\title{
Le livre déplacé, une bibliothèque hors-les-murs
}

\section{Julia Bonaccorsi}

\section{Résumé}

Il fait froid dans les bibliothèques qu'évoque Julia Bonaccorsi, les "bibliothèques de rues", ces lieux investis par des professionnels et des bénévoles pour porter le livre à la rencontre des populations défavorisées. Mais que devient le livre ainsi déplacé ? La médiation du livre abolit-elle ou renforce-t-elle les frontières culturelles ? Plutôt que d'évaluer la possibilité de franchir les distances sociales, Julia Bonaccorsi déplace la question. À partir de l'observation attentive à laquelle elle s'est livrée, dans la durée d'une action à laquelle elle a elle- même participé comme lectrice, elle montre comment se déploient et interfèrent au sien d'un même lieu les espaces multiples de la culture. Des extraits du journal d'observation sont cités au fil de l'analyse.

\section{Citer ce document / Cite this document :}

Bonaccorsi Julia. Le livre déplacé, une bibliothèque hors-les-murs. In: Communication et langages, $\mathrm{n}^{\circ} 127,1 \mathrm{er}$ trimestre 2001. Dossier : Le corps saisi par l'image. pp. 21-34.

doi : 10.3406/colan.2001.3057

http://www.persee.fr/doc/colan_0336-1500_2001_num_127_1_3057

Document généré le 15/10/2015 


\title{
Le livre déplacé, une bibliothèque hors-les-murs
}

\author{
Julia Bonaccorsi
}

II fait froid dans les bibliothèques qu'évoque Julia Bonaccorsi, les "bibliothèques de rues", ces lieux investis par des professionnels et des bénévoles pour porter le livre à la rencontre des populations défavorisées. Mais que devient le livre ainsi déplacé ? La médiation du livre abolit-elle ou renforce-telle les frontières culturelles? Plutôt que d'évaluer la possibilité de franchir les dis- tances sociales, Julia Bonaccorsi déplace la question. À partir de l'observation attentive à laquelle elle s'est livrée, dans la durée d'une action à laquelle elle a ellemême participé comme lectrice, elle montre comment se déploient et interfèrent au sien d'un même lieu les espaces multiples de la culture. Des extraits du journal d'observation sont cités au fil de l'analyse.

"Les lieux sont importants; aujourd'hui, des lectures publiques se font dans des lieux encore très " officiels", comme les bibliothèques. Nous souhaitons en sortir pour aller vers les publics les plus éloignés des livres, ceux qui ne font pas partie des réseaux culturels. "

Juliette Campagne ${ }^{1}$

" Il est parti devant le constat que le non-public existait puisqu'il ne l'avait pas trouvé."

Philippe Tiry ${ }^{2}$

Cet article trouve sa source dans une recherche menée en 19981999 autour d'une pratique de lecture marginale, la lecture de rue $^{3}$. II s'agit alors d'observer les spécificités de ces lectures en suivant les actions menées par une médiathèque de quartier à Lille, et dont le principe est que certains membres du personnel, aidés de bénévoles, lisent des livres à haute voix pour des

1. Juliette CAMPAGNE, «Lire à voix haute: dix ans déjà !", in Lire et dire, Actes des $1^{\text {res }}$ rencontres. 6-7-8 juin 1998, Chambon-sur-Lignon, Clermont-Ferrand, 1999, p. 34.

2. Philippe TIRY, « Un âne pour porter les prophètes ", Rue de la Folie, 8. 2000, p. 38.

3. Julia BONACCORSI, Étude d'une pratique de lecture contemporaine, la lecture de rue, mémoire de DEA dirigé par Annette BÉGUIN, université de Lille-III, UFR IDIST, 1999. 
enfants en se déplaçant au cœur même de leurs lieux de vie. Du point de vue d'un état des pratiques culturelles, la réalisation de la lecture à haute voix à l'extérieur de l'institution, dans la ville même, suggère un ensemble de questionnements éclairants sur la signification de cette pratique au $x x^{e}$ siècle. Le livre placé dans un contexte inhabituel, l'institution détachée de son cadre fondateur, les lieux et pratiques d'un cadre urbain confrontés à une initiative extérieure: cet ensemble de décalages volontaires ou subis, au cœur desquels s'inscrit la lecture de rue, dessine une image particulière et révélatrice des relations entre formes culturelles dans la ville d'aujourd'hui ; c'est ce que je voudrais mettre en évidence en explorant quelques aspects de ce jeu entre les livres et les lieux.

\section{UN PROJET DOUBLÉ D'UN PARI}

Retraçons très brièvement le contexte historique de ces séances de lecture «hors-les-murs». À la source de ces actions, on trouve le mouvement ATD Quart-Monde, qui crée des «bibliothèques de rue » dans les années 60 , avec l'objectif de lutter contre la pauvreté et l'exclusion, notamment par l'accès au savoir. Au début des années 80 , l'essor des politiques de lecture publique a suscité ce type de pratiques au sein même des bibliothèques municipales (opération dite « les bibliothèques hors-lesmurs »), souvent en partenariat avec le milieu associatif. Bénévole ou employé par la bibliothèque, le lecteur à voix haute de la bibliothèque hors-les-murs est porté par un projet doublé d'un pari; le projet de la médiathèque, institutionnalisé, mais aussi un pari personnel chargé de toute une idéologie: permettre aux enfants qui n'ont pas accès à la bibliothèque de connaître les livres, les « beaux livres ». Le contexte politique des années 80 , qui favorisait l'accès du plus grand nombre à la culture, a une résonance particulière dans le domaine du livre, car celui-ci apparaît comme porteur d'une symbolique forte, celle de la langue, de ce qui fait l'humain, dit Claudie Tabet ${ }^{4}$. L'idéologie sous-jacente aux actions s'inscrit dans cette logique du livre qui humanise, répare le tissu social, etc.

Je choisis de nommer ces acteurs de la lecture de rue des lecteurs, afin d'éviter toute interférence avec le terme « médiateur 
du livre ", qui correspond à une fonction dans les bibliothèques. Ainsi, dans le cas de ma recherche, une équipe de lecteurs bénévoles accompagne la lectrice médiatrice du livre de la médiathèque Lille-Moulins.

Précisons d'autre part que nous ne parlons pas ici des bibliobus mais d'une forme plus sommaire de déplacement: des lecteurs, venus avec une caisse de livres et quelques tapis de sol, prennent place sur le terrain de jeu d'une cité HLM, attendant que les enfants, après avoir choisi un livre dans la caisse, leur demandent de le leur lire. Dernière mise au point terminologique: je désignerai ces actions comme "lectures de rue ", réservant l'expression "bibliothèque de rue » au travail réalisé par les associations.

L'objectif de cet article est d'expliciter les dynamiques et les effets du déplacement de la bibliothèque de quartier par une mise en perspective des trajets et des confrontations d'espaces provoqués par cet acte de lecture. Je chercherai à éclairer ce que peut impliquer le déplacement physique d'une institution culturelle légitime dans un lieu désigné comme extérieur à cette culture, en décrivant les spécificités du contexte, les jeux d'influence et les mécanismes d'interférence entre le «lire » et l'« urbain ». Une telle analyse associe donc le livre comme objet culturel, la médiation culturelle comme engagement situé et la réalité des cultures existantes dans la ville.

Plusieurs trajets sont accomplis dans cette médiation, à la fois matériels et institutionnels, générant un déplacement d'un espace symbolique dans un autre. Le mouvement de recontextualisation du livre bouleverse la répartition des territoires, transformant des lieux, qui perdent plus ou moins les usages initiaux auxquels ils étaient destinés. Réciproquement, le trajet de la bibliothèque n'est pas sans incidence sur la performance de la lecture, la production par le lecteur. J'interrogerai d'abord les enjeux d'une telle «transplantation » d'un équipement institutionnel, avant d'analyser les jeux de territoires qui en découlent et les effets de cette pratique sur l'urbain.

\section{«Y ALLER »}

Dans cette recherche, le regard posé sur les actions de lecture de rue est interne aux actions elles-mêmes. En effet, j'ai mené le travail de terrain en observation participante, comme lectrice 


\section{Sociologie de la lecture}

bénévole de la médiathèque du quartier Lille-Moulins, au sud de la ville de Lille.

Arrêtons-nous un peu dans ce lieu : Lille-Moulins est un quartier populaire, divisé par l'ancienne ligne des fortifications (emplacement actuel du métro aérien) en deux zones: le «Vieux Moulins " (partie ancienne) et «Moulins-Belfort 》 (HLM des années 60). La médiathèque, construite dans une ancienne manufacture de textile du Vieux Moulins, doit donc, pour se rendre à Moulins-Belfort, traverser la frontière marquée par le métro aérien (frontière horizontale et verticale). Bien que très proche du centre, Belfort ne semble pas appartenir au quartier ni à la ville de Lille : l'uniformité de ses bâtiments, ainsi que leur disposition (tournés vers l'intérieur) en fait un lieu à part, qui forme un tout. C'est une architecture d'enclos, qui, au-delà de l'aspect " cage à lapins », est fermée sur elle-même.

II faut noter la densité de l'histoire du quartier de Moulins, qui a vu à partir du milieu du $\mathrm{XIX}^{\mathrm{e}}$ siècle plusieurs étapes d'urbanisation. Un nombre important de courées sont construites aux alentours de 1856, lors de l'implantation de manufactures de textile et de métallurgie, remplaçant les fermes et les jardins, ainsi que les moulins à huile qui ont donné leur nom au quartier. La récession des industries dans les années 50 font alors du quartier un « lieu refuge " pour les plus pauvres ; en fait, la médiathèque à l'intérieur du Vieux Moulins mène d'autres types d'actions (elle se rend aux Restos du cœur, à la PMI...).

Avec la lecture de rue dans Moulins-Belfort, il s'agit d'un déplacement d'une autre nature : c'est une frontière majeure de l'espace urbain contemporain qui est investie. La littérature sociologique et ethnologique propose de nombreuses descriptions de la discrimination des quartiers $\mathrm{HLM}^{5}$. La stigmatisation du lieu est visible dans l'expression «îlot Belfort », désignation usuelle du quartier, qui signifie bien la dissociation entre les HLM et le reste de Moulins. Parce qu'il est coupé du reste du quartier, et donc de la bibliothèque, celle-ci ne donne pas l'impression de lui appartenir et doit donc se déplacer elle-même. "C'est bien sur le mode d'un passage à l'acte, d'un engagement physique

5. Colette PETONNET, On est tous dans le brouillard, 1979 ; Claire CALOGIROU, Sauver son honneur, 1989 ; Jean-Marie DelaRUE, Banlieues en difficultés : la relégation, 1991 ; David LePoutre, Cour de banlieve, 1997. 
«sur le terrain » que se construit l'action ${ }^{6}$, dit Michel Péraldi à propos du DSQ (Développement social de quartier), « il n'est alors question ni d'établir des dispositifs, ni de mettre en œuvre des compétences et des logiques techniques, il faut d'abord y aller ». Ma recherche, nourrie des travaux réalisés en sociologie de la banlieue, peut dès lors partir d'une première hypothèse : la bibliothèque, en se rendant dans le lieu de vie du public qu'elle cherche à atteindre, superpose à l'espace physique (l'îlot Belfort) un espace social (le public cible). Ou encore elle réinterprète l'espace physique comme un espace social.

\section{UNE AMBASSADRICE DE L'INSTITUTION}

$Y$ aller, c'est non seulement aller dans la rue, mais s'inscrire au cœur d'un espace social dit en rupture. La distance à parcourir, au-delà du boulevard périphérique, est sociale et culturelle. II est éclairant de mettre ces trajets en perspective avec la « réserve prudente " et la peur du rejet stigmatisant que soulignent ceux qui ont analysé la non-fréquentation d'équipements culturels consacrés (en particulier Xan Bouzad ${ }^{7}$ ). Dans quelle mesure la bibliothèque hors de ses murs, extraite de sa carapace architecturale, instaure-t-elle (ou non) le même rapport avec les personnes qui se sentent dominées par la bibliothèque intra-muros? Dans ce mouvement «vers ", il y a quelque chose d'un apprivoisement: provoquer une rencontre entre des hommes et un objet de culture, par-delà le contexte institutionnel de la bibliothèque. Cependant, la visée ultime de ces lectures en plein air est d'attirer les «nonpublics » entre des murs plus légitimes; la lecture de rue est souvent nommée «médiatrice». Mais ce terme réveille trop de polémiques sur son sens même : je la dirais plutôt ambassadrice de l'institution. Gardons à l'esprit cette mission (parmi d'autres : le plaisir du texte, l'accès à un patrimoine culturel, etc.) comme un des fils qui tissent notre réflexion.

Première conclusion : la lecture de rue se situe dans une logique des rapports entre proche et lointain, dans une dialectique de la distance constamment en tension. Distance à la fois matérielle et

6. Michel PERALDI, "Le Cycle du fusible. Jalons pour une histoire sociale du DSU à Marseille ", Annales de la recherche urbaine, $n^{\circ} 68-69,1995, \mathrm{p} .75$.

7. Xan BouZADA, «La Distance sociale et symbolique aux équipements culturels", in Jean-Pierre Augustin\& Daniel LATOUCHE (dir.), Lieux culturels et contextes de villes, Talence, Maison des sciences de l'homme d'Aquitaine, 1998, pp. 63-72. 
symbolique : l'analyse que fait Georg Simmel des organisations spatiales de la société est une base importante pour une réflexion sur l'espace et l'action ${ }^{8}$.

Pour Simmel, "l'imagination qui travaille dans l'éloignement déchaîne une exagération effrénée de sentiments à côté de laquelle les excitations de la proximité physique [...] paraissent néanmoins connaître une certaine limite et mesure. ${ }^{9}$ " La proximité, parce qu'elle n'est pas toujours désirée, peut entraîner des contraintes, mais elle peut aussi être très positive. Elle est en tout cas, selon Simmel, préférable au dérèglement imaginatif qu'engendre l'éloignement: le «pathos de la distance» (Hall), la réserve prudente face à la culture légitime et les équipements qui la symbolisent.

\section{ESPACES AMBIGUS}

\section{La « rue » de la lecture de rue}

L'expression elle-même impose secrètement, au niveau littéral, la connotation d'une pratique à caractère non conventionnel. La «lecture de rue " se distingue de la «lecture publique » qui désigne les lectures à haute voix réalisées dans des espaces métamorphosés, comme les librairies, les cafés, les bibliothèques, etc. : la « rue » ici convoquée renvoie indiciblement (audelà de la sonorité du terme) aux arts de la rue et à l'imagerie qui en découle (spectacles clandestins, cracheurs de feu, saltimbanques...). Ce n'est pas la rue dans un sens géographique, mais plutôt dans un sens légendaire, qui va référer au populaire, au marginal, au non-légitime, en particulier à ce théâtre qui jaillit dans les années 70 . La bibliothèque hors-les-murs qui produit cette lecture de rue ne peut être indifférente à ces représentations, elle doit composer avec elles, matériellement et symboliquement. Mais par-delà ce fond de citation plus ou moins mythique, quelle est en l'occurrence cette rue?

8. Georg SIMMEL, Sociologie. Etudes sur les formes de la socialisation, Paris, PUF, 1999 ; chap. 9: «L'espace et les organisations spatiales de la société », pp. 600-684.

9. Ibid., p. 628 


\section{Les lieux de l'observation}

Le groupe de lecteurs se rend dans le même après-midi dans deux rues de l'îlot Belfort, ce qui a engendré une observation en deux volets : la rue Delesalle et la rue Herriot. La rue Delesalle est un espace délimité par quatre HLM, formé d'un espace central qui est le terrain de jeu des enfants et d'une voie qui permet aux voitures de circuler et de se garer devant les immeubles. Ainsi, en quelque sorte, cet espace au milieu des immeubles joue le même rôle qu'une place, à la fois lieu de passage et lieu d'arrêt, de repos; dans le Vieux Moulins, la place Vanhoenacker est un espace de sociabilité très important pour le quartier. Cependant, cette «place » rue Delesalle n'est pas vraiment publique ; en fait, elle appartient par sa position centrale au groupe d'immeubles; elle ne possède en effet qu'une entrée pour les voitures, et quelques passages pour piétons: un sas d'entrée.

Rue Herriot: l'espace de la lecture est situé devant un immeuble ; devant, la rue et, au loin, de l'autre côté de la rue et de pelouses en travaux, le boulevard Belfort, frontière aussi haute que large. Cependant, la rue Herriot a une fonctionnalité similaire à la rue Delesalle : accès vers les immeubles, elle est aussi un lieu de rencontre, où se tiennent parfois des habitants. Elles sont des espaces de transition semi-publics car elles restent des accès aux parkings (et non pas des voies de circulation). En plein air, sans barrières apparentes, les espaces situés rue Delesalle et rue Herriot sont ouverts et de libre accès.

\section{Une vie de quartier...}

Pour des raisons architecturales et matérielles, la lecture de rue va donc s'inscrire dans un cadre singulier, mi-public, mi-privé, lieu de transition entre l'extérieur et «l'îlot », et qui est le théâtre quotidien des rencontres et des échanges entre les habitants des immeubles.

L'espace investi par la lecture est-il un espace privé ou public? « II est quelquefois difficile de déterminer avec précision ce qui est espace privé et ce qui est espace public, remarquent Edmond Marc et Dominique Picard, car cela dépend en grande partie de l'activité que l'on y exerce, des gens avec lesquels on s'y trouve et de la façon subjective dont on l'investit. ${ }^{10}$ " Les auteurs donnent l'exemple d'un wagon de chemin de fer qui devient privé quand une famille s'y trouve seule et occupe entièrement le lieu. Ces lieux de passage, s'ils n'ont pas le caractère 
anonyme des places publiques, s'organisent en différentes zones d'utilisation, celles-ci pouvant parfois se mêler. Les pratiques des habitants ont en quelque sorte dessiné un schéma virtuel, mais opérant des espaces qui contextualisent la lecture de rue. Plusieurs types d'activités se déroulent dans ces espaces: les enfants jouent; des groupes de jeunes discutent; c'est aussi un lieu de promenade; après les courses, les parents vident le coffre de leur voiture, les enfants venus en renfort les retrouvent devant les entrées des immeubles. Au sujet des espaces d'usage public, Guy Barbichon parle d'espaces partagés, dont les fonctions peuvent être simultanées ou successives, et signale «la variation des positions, d'occupation et d'appropriation des usagers " 11 . C'est à partir de cette conception vivante et pratique de l'espace socialisé que le schéma initial du dedans-dehors va pouvoir être enrichi et dépassé.

Le quotidien et l'exceptionnel de la vie familiale prennent place dans ces espaces-sas d'entrée. Tous les samedis, un vendeur de légumes fait la tournée du quartier avec son tracteur, les habitants sortent faire des achats. Comme je l'ai signalé tout à l'heure, c'est là que se vident les coffres des courses; c'est là aussi que se préparent les événements exceptionnels :

"C'est le jour de l'aïd. Les enfants sont tous dehors, très bien habillés, en attente de la fête. Ils sont très excités. Les habitants s'agitent devant les immeubles, un homme rapporte sur son épaule une énorme sac-poubelle: il contient le mouton, les enfants s'agitent, l'un d'eux part en courant rejoindre l'homme. "

(27.3, rue Delesalle ${ }^{12}$ )

Ceci montre bien le caractère sommaire du premier modèle invoqué, la bibliothèque comme lieu culturel légitime pénétrant dans un espace qui lui est étranger. Dans cette atmosphère, le lecteur est confronté à l'existence d'une culture. II a la sensation de pénétrer dans un lieu clos, intime, et de participer par le regard à la vie privée des habitants. Tous les enfants ne fêtent pas l'aïd, et pourtant ils sont tous concernés par la fête ce jour-là, touchés par l'excitation générale.

11. Guy BARBICHON, «Espaces partagés : variation et variété des cultures », Espaces et sociétés, $n^{\circ} 62-63,1990$, p. 111.

12. Julia BONACCORSI, extrait du journal de terrain, 27 mars 1999. 
La famille se manifeste périodiquement dans le cadre de la surveillance des enfants. D'une part, les parents ont par les fenêtres un point de vue sur la place, ce qui leur permet de laisser les enfants sortir seuls. D'autre part, les enfants viennent souvent en phratrie sur la place. La présence des aînés, mais aussi des frères et sœurs plus jeunes, va interférer fortement sur la réception de la lecture, et parfois même sur la performance du lecteur. Lappropriation habituelle de l'espace se fait par l'occupation physique dans la rue (réunion autour des voitures, sur les perrons d'entrée, voitures laissées en double file devant la porte...), mais aussi par la voix (on s'appelle d'un bout à l'autre de la place, du haut des immeubles...) et par le regard englobant que les habitants ont depuis chez eux. Plus qu'il n'exerce une simple surveillance, ce regard enveloppe l'espace entier où se réalise l'action-lecture. Les habitants sont alors l'unique public de cette grande scène qui réunit divers espaces : le terrain de jeu, l'action lecture et la rue. D'une certaine manière, la lecture est subordonnée à ce regard. Si l'on prend la peine de regarder de ce point de vue large, la fiction d'une simple action-lecture se mue en rencontre, dans un espace, d'un ensemble emboîté de perspectives culturelles.

J'ai évoqué la voix: crier dans un lieu public pour appeler quelqu'un est un comportement rare et ponctuel; dans un lieu anonyme, deux personnes très éloignées n'engagent pas une conversation en haussant la voix (en Occident du moins). En revanche, au sein d'une maison familiale, ce type d'interaction est récurrent. L'environnement de l'action-lecture, traversé par ces voix, devient au même titre qu'une maison un lieu du familier, de l'intime. Le regard et la voix concourent à signifier une symbolique de ces espaces de transition entre la ville et l'îlot Belfort: ils sont devenus la propriété des habitants, dans un sens immatériel, impalpable, mais qui n'est pas sans incidence sur la place et le statut de la bibliothèque hors-les-murs.

À l'intérieur de ce cadre général, la bibliothèque choisit de s'installer sur les terrains de jeu, espace privé des enfants, reproduisant, consciemment ou non, le choix des dames de l'école du dimanche au $X \mathrm{~V} I I^{e}$ siècle. Scolarisés au même endroit, voisins, ces enfants se connaissent, ils ont l'un pour l'autre figure d'habitués. Ils ne se déplacent pas d'un groupe d'immeubles à l'autre : ils restent là où les familles peuvent avoir un regard sur eux. 


\section{...et la lecture de rue}

La bibliothèque hors-les-murs constitue un investissement second, spectaculaire et rituel de cet espace déjà secrètement structuré par le rythme des pratiques sociales. Elle s'installe toujours au même endroit, selon la même disposition. Les lecteurs s'assoient sur les bancs ou sur les murets pour des raisons pratiques évidentes ; d'autre part, la lecture de rue est fondée sur la régularité : régularité du jour de venue et de l'horaire. L'utilisation des tapis de sol comme isolant sur les bancs et les murets donne un premier signe d'une délimitation de l'espace; ils ont une fonction de marquage visuel, de bornes pour un lieu de lecture. L'installation de la bibliothèque n'implique pas que l'espace soit vide, les lecteurs ne chassent pas les habitants mais cherchent plutôt à engager une conversation, à s'en faire connaître.

De la même manière, la bibliothèque hors-les-murs, en investissant une partie d'un territoire donne un nouveau sens et une nouvelle fonction à ce territoire, et ceci de manière chronique : à l'inverse du théâtre de rue, ponctuel et éphémère, l'appropriation de la lecture de rue est répétitive.

D'une certaine façon, quand la caisse de livres est là, symbole de la bibliothèque, tous les éléments de l'espace préexistant sont censés être captés par un usage, celui de l'action-lecture. Lors de l'exploitation de mon terrain, j'ai défini comme « foyers d'attention » l'éclatement de l'action-lecture en petits îlots centrés autour d'un livre et d'un lecteur (mais il peut y avoir plusieurs enfants). Ces foyers peuvent être perméables (par exemple lorsqu'un chant attire les autres enfants) ou très fermés (selon la position du petit groupe, sous un parapluie par exemple). L'espace de ces foyers est investi par la voix du lecteur (qui doit couvrir celle des autres lecteurs), la gestuelle, le livre, tenu loin devant pour permettre à tous de voir, etc. Mais aussi systématique qu'elle soit, cette pratique culturelle ne devient pas hégémonique, cet espace importé n'efface pas l'espace investi. Tout juste y laisse-t-il une trace analogue à celle que laisse sur le sable le mouvement intermittent de la mer.

\section{INTERFÉRENCES}

En me bornant ici aux observations relatives à deux fonctions précises des espaces publics, le jeu et la lecture, je vais tenter de décrire la plasticité de l'espace et la mobilité des territoires. 
Parce que la lecture de rue se positionne dans (ou à proximité de) l'espace consacré au jeu, celui-ci est sans cesse suggéré, rappelé dans l'espace de la lecture. Cette interférence prend un ensemble de formes, donnant matière à variations.

"Le groupe est sans cesse en mouvement. Des fillettes jouent à l'élastique entre les tables, mais seulement deux d'entre elles participent à la séance. » (6.3, rue Delesalle)

"Des petites filles jouent à la corde très près de la table. Il s'agit d'un jeu très organisé, avec des tours de passage. Elles agrandissent encore la corde en la nouant avec une autre corde. Anna lit et chante avec moi sur une table de ping-pong. Elle ne peut résister à l'appel du jeu et rejoint ses camarades. ”

(12.6, rue Delesalle ${ }^{13}$ )

Dans un premier cas, certains enfants viennent seulement lire, ils rentrent chez eux après, ils ne jouent pas dehors.

" Une fillette est appelée par une fenêtre, elle est accompagnée de sa sceur. Une troisième fillette, très au courant, m'explique qu'elles n'ont pas l'habitude de sortir. » (13.3, rue Delesalle $\left.{ }^{14}\right)$

Le jeu est alors un simple élément, une pratique extérieure : l'enfant et le lecteur ont le même rapport au jeu, il ne les concerne pas. Bien entendu, les enfants peuvent être attirés par cet espace, à la fois par la configuration matérielle mais aussi pour ce qu'il signifie (défouloir...), mais il leur reste étranger, même s'ils connaissent d'autres enfants.

Dans un second cas, la participation à la séance de lecture est complète ou presque, le jeu vient après (ou avant) : l'enfant dissocie les deux espaces, espace du jeu et de la lecture, d'un point de vue temporel du moins; il y a pour lui temps de la lecture et temps du jeu. C'est souvent le cas des enfants les plus âgés.

Enfin il arrive, dans un troisième cas, que le rapport jeu et lecture soit fondé sur l'alternance. L'enfant change d'activité, et par cela même la fonction de l'espace se trouve modifiée. Par exemple, l'enfant peut être accaparé en cours de lecture par des camarades qui cherchent à échanger des «Pog's " avec lui : pendant quelques minutes, sur le banc auparavant consacré au livre, l'attention va être centrée sur le jeu, la lecture restant malgré tout

13. Julia BonACCORSI, extrait du journal de terrain, 12 juin 1999.

14. Ibid., 13 mars 1999. 
suggérée par le lecteur et les livres, ainsi que par le tapis de sol. Le jeu se substitue à la lecture, mais ne la remplace pas intégralement. Ce phénomène d'alternance entre les deux espaces n'est pas seulement produit occasionnellement par le va-et-vient des enfants d'une activité à l'autre, il est rappelé en permanence par certains éléments, comme un vélo couché sur le sol devant le banc, ou encore l'arrière-plan que manifeste une scène comme celle-ci :

"Elle tient un ballon sous le bras. II tombe souvent sur le sol et elle doit à chaque fois aller le chercher, elle le fait rebondir. Je lui demande de le ranger dans un coin, en attendant. "

(13.3, rue Herriot ${ }^{15}$ )

Ici, le ballon est une transition entre les deux espaces. Le jeu est en attente, mais la chute perpétuelle du ballon et le bruit qu'elle engendre intervient dans la concentration de la petite fille et des autres enfants.

Ces éléments, rappel du jeu, ne provoquent pas forcément, comme dans le cas du ballon, une interférence pouvant faire obstacle à la lecture : ils sont parfois simplement présents, ainsi les patins à roulettes aux pieds des enfants...

Deux fonctions sociales pour un seul lieu? L'espace du jeu et l'espace de la lecture, reliés par les mouvements des enfants, sont imbriqués l'un dans l'autre, à tel point qu'ils ne se distinguent peut-être pas dans le vécu que les enfants ont de leurs gestes.

On est ici loin de ce qui serait une simple acculturation. Selon une expression de Simmel, l'espace se « remplit 》 des actions réciproques entre les hommes, de la socialisation ${ }^{16}$.

Ces territoires, à l'intérieur de l'espace public, ont une épaisseur forte puisqu'ils présentent plusieurs niveaux d'analyse: à l'espace physique, matériel, investi par la lecture de rue, se superpose un espace symbolique (Livre, Culture, Savoir) qui à la fois lui correspond et déborde ses limites physiques; à l'espace architectural des HLM est associé un espace symbolique dense, puisqu'il convoque à la fois l'histoire locale du quartier et les

15. Ibid., 13 mars 1999.

16. Georg SiMmeL, Sociologie, op. cit., p. 601. 
images d'une banlieue fantasmée (absence d'identité, de culture, etc.). Ces espaces s'entrecroisent et se nourrissent les uns des autres sans qu'il y ait jamais rupture ni espace propre (et neutre) pour la lecture, à la manière de papiers calques qui laissent transparaître ce sur quoi ils sont posés.

\section{Signes de l'autre}

L'espace de la rue, espace de vie, n'est donc pas gommé par l'installation de la bibliothèque hors-les-murs ; au contraire, il est sans cesse signifié par la présence d'éléments qui lui sont propres. Le bruit, occasionné par le haut-parleur du tracteur de légumes, par exemple, qui envahit la rue entière est un trait signifiant d'un espace de vie, de même que les capsules de bouteilles de bière laissées sur le sol. Les habitants redessinent constamment par certains de leurs actes les bords de leur territoire de vie : en lançant les blousons des enfants par les fenêtres, en s'interpellant...

Ce sont, pour les militants et les employés de l'« action culturelle », autant de signes de l'autre, de l'existence d'une culture autre, autant de rappels pour la bibliothèque hors-les-murs qu'elle est de passage. Dans un lieu unique, celui de la rue, se produisent des interférences entre les espaces qui le remplissent (en prenant en compte la distinction que propose Jean Caune: "Le lieu est une configuration de dispositions qui organise un ordre et implique une stabilité. L'espace, lui, est animé par les mouvements humains qui s'y déploient. ${ }^{17}$ "). La lecture de rue s'ouvre alors à ce qui constitue peut-être sa réelle dimension culturelle, en manifestant la rencontre et la tension matérielles, temporelles et spatiales entre les définitions mêmes de ce qu'est une culture et en questionnant l'idée même d'une coupure entre la culture et la vie.

En s'appropriant un territoire dans l'espace de la rue, la lecture de rue réalise une combinaison d'activités. Son caractère incongru est diminué par cette combinaison, ce mélange, qui fait du groupe formé par les lecteurs et la caisse de livres une manifestation familière. 


\section{Sociologie de la lecture}

La localisation de la bibliothèque hors-les-murs dans un espace public - ce qui est une délocalisation de la bibliothèque - n'est pas une appropriation exclusive d'un territoire. Cet espace public, en tant qu'espace social, resurgit sans cesse à la manière d'un fond visuel et sonore. Sur cet arrière-plan spatial et temporel, l'espace de lecture va se répartir en unités plus petites, en foyers d'attention : ces groupes proposent aux habitants le spectacle de la lecture de rue, les habitants formant alors son public.

À la lumière de ce terrain et au terme de la réflexion, on entrevoit la grande complexité qui singularise ce type d'action, trop souvent résumé à grands traits manichéens. La tension constante entre les pratiques en contact dans ce lieu laisse des empreintes de manière réciproque pour les habitants et l'institution.

Julia Bonaccorsi 